\title{
Evaluative language in physiotherapy practice: how does it contribute to the therapeutic relationship?
}

\begin{abstract}
In physiotherapy, the therapeutic relationship - in which a therapist and patient work together to achieve treatment goals - is increasingly seen as the foundation of patient care. How the therapeutic relationship is established and enacted, however, is not well understood. One way to better understand the nature of the relationship is to examine how therapists and patients evaluate and inform each other about the patient's physical capacity, sensation, and emotions. As the patient and therapist's talk is the primary means to realise and exchange such evaluations, our focus is on evaluative language used by the therapist and patient in their interactions. The aim of this paper is to examine the language and function of evaluation in physiotherapy consultations.
\end{abstract}

The study is a discourse analytic one using Appraisal Theory. In Appraisal Theory, language resources that speakers use to construe evaluations such as emotions, judgments of behaviour and aesthetics are expressed as a system. The sub-systems are AfFECT (expressing emotion), JUDGMENT (assessing behaviour) and APPRECIATION (evaluating processes and objects). The data are a convenience sample of 18 consultations from two cultural and therapeutic settings: primary healthcare (Sweden, Australia); and hospital rehabilitation (Australia). The findings show that both patient and therapist utilise all sub-systems of Appraisal; however, use of the sub-systems by the therapist and patient differs functionally. JUDGMENT and APPRECIATION play a central role in therapists' co-construction of patients' physical history and presenting problem. In contrast, patient AfFECT evaluations, mainly to do with emotions about loss of capacity and pain, are 
generally not followed up by the therapist. The findings suggest that while patients engage with the therapeutic relationship from a clinical and interpersonal perspective, therapists are more narrowly focused on their own clinical tasks. The study findings have implications for understandings of the therapeutic relationship in physiotherapy and can inform teaching.

Keywords: Australia, Sweden, physiotherapy, therapeutic relationship, discourse analysis, communication, clinician-patient interaction, patient-centred care 


\section{Introduction}

\section{Evaluative language in co-constructing physical therapy}

Physiotherapy is a major healthcare provider with treatment involving the active engagement of both patient and physiotherapist to achieve desired therapeutic outcomes (Poulis, 2007). Physiotherapists assess patients' mobility and capacity. They provide and facilitate treatment through verbal and physical interaction with patients, activating patients' inherent resources (Broberg \& Tyni-Lenné, 2009). During therapy, physiotherapists evaluate patient performance, providing both positive evaluations of patient performance, for example, "lovely", as well as verbally providing corrective feedback on performance errors (Parry, 2005). The manner in which physiotherapists respond to patients' physical performance and the words and phrases they use can have implications for patients' learning and future performance (Martin \& Sahlström, 2010) as well as for the relationship between therapist and patient. Patients in turn participate in the interaction with the physiotherapist but not only as recipients of treatment: they can have agency to monitor, inform, and self-evaluate. For example, therapists' support of patients' selfmanagement strategies is highly valued and has been shown to be more effective than specific interventions for lower back pain (Balagué et al., 2012). Such support may be visible through the therapists' language choices such as their verbal evaluation of healthy or unhealthy behaviour (Josephson \& Bülow, 2014). The language used by both the therapist and patient plays a key role in co-constructing the physiotherapy interaction, the treatment relationship, and the joint achievement of treatment goals, with evaluative language appearing to play an important role to express feedback on performance of exercises, physical capacity, and sensation. 
The ways in which language shapes the physiotherapy interaction can be examined in the broader context of contemporary physiotherapy practice and its espoused values including patient-centred care. Key dimensions of patient-centred care are: adopting a biopsychosocial perspective; demonstrating respect for the patient; sharing information, responsibility, power and decisionmaking; and prioritising the therapeutic relationship (Mead \& Bower, 2000). Patient-centred care is accepted and promoted by the physiotherapy profession as being the cornerstone of interactions with patients (e.g. Beeston \& Simons, 1996; Harman et al., 2011; Jensen et al., 2000; Pinto et al., 2012; Potter et al., 2003); it is also endorsed by physiotherapy practice guidelines (Broberg \& Tyni-Lenné, 2009; APA, 2011; CSP, 2014). A patient-centred approach to care contrasts with a biomechanical approach that is seen to separate mind and body (Thornquist, 1994), potentially limiting the physiotherapist's ability to achieve aspects of patient-centred care (Mudge et al., 2014). In contrast to a patient-centred approach to care, in the medical literature the biomedical approach is considered to be practitioner-centred, with the patient a passive recipient of treatment (Wade \& Halligan, 2004).

Despite the promotion of patient-centredness, there is minimal empirical research to inform whether patient-centred concepts are achieved in physiotherapy practice. Early work by Thornquist (1994) investigating how physiotherapists relate to and examine patients identified a dualistic frame of reference in which therapists' prioritised their biomechanical frame of reference over the patient's experiences and perceptions, suggesting both a discrepancy and lack of alignment between therapist and patient concerns. Thornquist points out that the different frames of references in her findings resonate with Mishler's 'voice of medicine' of the therapist and that of the 'lifeworld' of the patient (Mishler, 1984). A recent paper argues that the physiotherapist perspective of patient-centred care and what this means in clinical practice is not 
well understood (Cruz et al., 2012). Eisenberg (2012) argues for an alternative approach in which the physiotherapist in conjunction with the patient looks beyond the therapist role to "let go of assumptions about what is "best'“ (page 445). Such an approach would allow the therapist and patient to explore meaningful options even if the outcomes diverge from typical rehabilitation goals and assumptions of 'good' outcomes. This could shift the balance toward a more equitable interaction in which the patient's perspective is more deeply incorporated (Eisenberg, 2012).

The therapeutic relationship has been considered as a non-specific element, facilitating other components in patient-centred care (Sidani \& Fox, 2014). Sidani and Fox describe this element as a relationship in which trust and nurturing is foundational, respect is mutual, and information that will guide the planning, implementation and evaluation of care is exchanged by both parties. Mutual respect is interpreted as meaning that patients respect the professional's expertise, and that healthcare professionals are open to and respectful of patients' knowledge and experiences. To enact these elements of the therapeutic relationship, healthcare professionals need competent communication skills, including a capacity to explore patients' concerns and preferences (Sidani \& Fox, 2014). The term therapeutic is commonly associated with an outcome, that is, an intervention that influences the patient in a certain direction, and that this direction has a meaning for the patient's understanding or recovery. A review article of the therapeutic relationship in physiotherapy points out that some aspects are well addressed in the themes of congruence, partnership, and physiotherapists' roles and responsibility but poorly addressed in the themes of communication, and personalised therapy and other relational aspects (Besley et al., 2011). In this paper, we conceptualise the therapeutic relationship as contributing to patient-centred care in accordance with Sidani and Fox (2014) and concur that how therapy is personalised and the relational aspects played out warrants further investigation. Further, as with patient-centred care, 
there appears to be limited empirical evidence as to whether and how these concepts are achieved in physiotherapy practice.

One way to gain insights into the therapeutic relationship in physiotherapy and how it is enacted is through the lens of language. Examining how interactants use language to navigate relational aspects such as mutual respect for each other's expertise, and engagement with patient preferences and experiences can inform not only the relationship construct but also provide evidence of gaps between the espoused approach and practice. This paper's aim is to examine how therapists and patients evaluate aspects such as physical capacity, sensation, and emotions within the physiotherapy consultation. We refer to these aspects overarchingly as evaluation, after Martin and White (2005), and examine evaluative language as its expression in the therapeutic interaction.

Our research question is: How is evaluation used by therapists and patients in physiotherapy consultations? We also investigate the alignment of evaluations used by therapists and patients; that is, whether there was agreement about the appraisal and whether there was uptake or acknowledgment of an appraisal by the listener (patient or therapist). This latter focus builds on Thornquist's observation (1994) of the discrepancy between the therapists' and patients' frame of reference.

\section{Methodological framework}

The ways in which therapists and patients use evaluation in physiotherapy consultations is examined using Appraisal Theory (for example, Martin, 2000; White, 2004; Martin \& White 2005). Appraisal Theory is a form of discourse analysis that has developed within systemic functional 
linguistics in order to examine and explain the ways in which speakers and writers express reactions such as attitudes and emotions, both positive and negative. In Halliday's systemic functional linguist (SFL) theory (for example, Halliday 1978) an underlying principle is that of choice, with language viewed as a system of meaning potential organised to make three main types of meanings: experiential meanings (representational), textual meanings (how content is organised), and interpersonal meanings (roles and relationships). Appraisal theory extended the model of interpersonal meanings to express as a system the enormous amount of lexical and grammatical choices that speakers use to construe evaluations such as emotions and attitudes, judgments of behaviour and aesthetics (Martin \& White, 2005). The system refers to a set of options that are available to the speaker to express meanings (and the linguistic means of expressing them) that are typically used in particular contexts (Martin, 2000). The sub-systems are AFFECT (language resources for expressing emotion), JUDGMENT (assessing behaviour) and APPRECIATION (making judgments about processes and objects) (Martin \& White, 2005). In systemic functional linguistics, language systems are conventionally written in capitals. Throughout this paper, we use initial capitals only for the Appraisal sub-systems. Appraisals can be expressed adjectivally (I felt frustrated), adverbally (Sadly, I can no longer ski), verbally, (He fears another back injury), and nominally (My wish is to run again). They can be single words that express a writer's or speaker's attitude towards some entity, but also phrases, word combinations, and stretches of language that convey a proposition or attitude (White, 2004): for example, really beneficial; Not as much as I should have been. Appraisals can also be implied, requiring contextual knowledge such as knowledge of technical language or slang in order to interpret the positive or negative appraisal of the speaker/writer. In spoken language, they can also be evident in the voice quality such as in tone or emphasis. 
Evaluation of evaluative language in discourse is an established area of inquiry in applied linguistics (Sarangi, 2003), yet the majority of approaches focus on a particular dimension of evaluation such as stance (Hunston \& Thompson, 2001). Our choice of Appraisal Theory was to address our research question was motivated by the theory's elaborated and systematic approach to dimensions of evaluation that resonated with our combined datasets; for example, patients' expression of emotion [Affect], and therapists and patients' evaluations of behaviour [Judgment]. Informed by a theory of language in context (SFL) that sees language as structured for use, Appraisal Theory is relevant and transferable to the clinical setting. Appraisal Theory has been used extensively to examine expression of opinions and attitudes in media texts, argument in secondary and academic writing, interplay of voices in legal discourse and in everyday social interactions (see Martin \& White, 2005 for an overview), yet to the best of our knowledge, it has not yet been applied to professional interactions in health settings. While conversation analysis (CA) is the dominant discourse analytic approach in healthcare communication research, it is not suited to systematically address the questions proposed in this study. How we have adapted Appraisal Theory to the physiotherapy setting is explained below.

\section{Method}

\section{Study genesis, design and data}

Our inquiry into the function of evaluation stems from initial discussions at an international meeting in 2011 at which two of the authors presented papers about physiotherapist-patient communication (IJ, RWK). While the focus of the presentations was not engagement, we recognised patterns in each others' data and acknowledged the potential empirical research value in combining the clinical and broader professional insights of physiotherapy, health professional education,, and applied linguistics. We therefore explored the phenomenon of evaluation using 
existing data that are convenience samples and that are from diverse physiotherapy treatment and clinical settings. The study utilises three data-sets: one from primary care in Sweden, the other two from Australia with one study conducted in a teaching hospital and the other in private practice. The three original studies broadly investigated communication between therapist and patient using interactional data: the Swedish data were part of a PhD study examining decision making about treatment for patients with lower back pain (Josephson et al., 2013); the Australian study in the teaching hospital focused on physiotherapists' perspectives of effective communication between student and patient as discussed in their feedback to students (Woodward-Kron et al., 2012); the Australian study in private practice examined the process of gaining informed consent (Delany, 2007). The researchers are physiotherapists (IJ, CD, AH), health professional educators (RWK, CD), and an applied linguist embedded in the health professional context (RWK). All authors are experienced qualitative researchers.

This study is a discourse analytic one, using the methodological framework of Appraisal Theory (primarily Martin \& White, 2005) to explore the phenomenon of evaluation in physiotherapy interactions. The discourse analysis was guided by RWK, whose PhD utilised the theory of language informing this study (systemic functional linguistics) and which incorporated Appraisal Theory. A total of 18 physiotherapists with an additional 8 supervising therapists, and 18 patients participated in the three studies: eight of these therapists were final year physiotherapist students whose supervisors were present in the interaction and who provided some verbal input. Table 1 provides demographic information on the participants as well as the type of clinical presentations and treatment focus. Information about whether the patient's visit was the initial visit or a recurring visit was not available for all data; therefore this has not been included. 
Each study had approval from the ethics committees in their respective study settings: Swedish data: The Regional Ethical Board in Linköping, Sweden, Dnr M194-08; the Australian hospital data, Austin Health Non-Drug Study Ethics Committee, N0409; and the private practice Australian data, School of Population Health departmental ethics committee at the University of Melbourne, DPH 1/2003. In each study, the project was explained verbally and in writing via a participant information form to potential participants: each project was outlined as investigating facets of communication between therapists, including student therapists, and patients. All participants signed a consent form. Recruitment processes are detailed in the original studies (Josephson et al., 2013; Woodward-Kron et al., 2012; Delany, 2007). No participants withdrew from any of the studies. The researchers accessed each others' data as de-identified transcripts in accordance with original ethics approvals. 
[insert Table 1 about here]

The Swedish data were video-recorded while the Australian interactions were audio-recorded. The total interaction time of recorded data is over 8 hours. The researchers were not present during the audio or video recordings of the interactions; at the Swedish site, the first author collected the data and consented participants; at the Australian private practice setting, the third author collected the data and consented participants; and at the Australian hospital setting, a research assistant collected the data and consented the participants. In the three studies, none of the researchers or research assistant had any direct professional relationship with any of the participants.

\section{Data analysis}

Audio-recordings were transcribed including overlaps and pauses, and the Swedish data were translated into English for the purposes of this study. Emphasis of words was noted in the transcripts as well as heightened emotional aspects such as laughter or sadness.

Two researchers (authors 1\&2) working independently identified the evaluations in each data subset, deductively sorting the identified appraisals to the sub-systems: Affect, Judgment, Appreciation, as described by Martin and White (2005). The inclusion criteria for the evaluations were evaluations directed towards: the patient as a person; the patient's health problem; or the patient's behavior and feelings. The exclusion criteria were: therapists' self-evaluations, and evaluations about waiting room matters. Each appraisal was coded once. The identified appraisals were then further analysed in regards to their communicative and treatment focus, such as assessment of physical function. This step involved two researchers comparing the analyses until agreement was reached. This verifying and decision making process was aided by the researchers' contextual interpretations of participants' use of evaluations. The researchers developed a coding 
book to guide and verify the analysis (Table 2). The left column of the coding book shows an outline of the sub-system components as elaborated by Martin and White (2005) (for language samples to illustrate these elements, see Martin \& White, 2005). The middle column shows the adaptation of this sub-system to the context of physiotherapy. To do this, we conflated the components for each sub-system from the left column and used descriptive labels that resonated with aspects of physiotherapy patient-discourse, for example, 'motivation' as part of Affect for the treatment /goal-setting aspect of physiotherapy-patient talk. The third column shows examples from the data to illustrate the contextual interpretation. Appraisals for each data set were tabulated according to the sub-systems with the appraiser (the source of appraisal) noted, and the contextual interpretation recorded.

As the analysis sought to identify how evaluative language was used by both physiotherapist and patient and how these evaluations functioned, the analysis was primarily qualitative; hence, the findings have not been quantified.

[Insert Table 2 about here]

\section{Findings}

The findings for the evaluation analysis are reported sequentially for the three sub-systems Affect, Judgment, and Appreciation.

\section{Affect [emotions]}

In the physiotherapy interactions, Affect appraisals functioned for the interactants to exchange information about the patients' in/security, inclination (desires and fears), un/happiness, motivation, as well as dis/satisfaction. One discourse pattern in which Affect appraisals occurred 
was when therapists enquired about patients' emotional state at the start of the consultation. The therapists' used a general question How are you feeling? to elicit patients' holistic self-assessment in terms of his/her emotions in relation to their treatment and continued treatment that was likely to cause the patient pain. The patients' responses, such as all right; not too bad in turn provided a holistic assessment. Similarly, during physical treatment as part of the consultation, such as ambulation in a hospital ward, or at the completion of a functional assessment, the therapists inquired of the patients' emotional state; in these instances, the focus was on patients' in/security, for example, good, so far. The therapists tended to repeat patients' responses for confirmation. The therapists' question, How does it feel? was also asked during physical tests. In this context, we propose that the question functioned to guide patients' responses towards their bodily reactions to the physical test, that is, an Appreciation (quality) rather than Affect (emotion) appraisal, to inform the therapists' diagnosis. Patient responses to this question, however, could reflect emotion such as fear: I am scared how to do it and not get hurt (SW5).

The Affect appraisals above were made in the context of giving and seeking information, that is, in the exchange structure of question and response. Appraisals also occurred in declarative statements by patients, for example, to express a desire or wish, I want to play netball (H04); to run again (SW1), or simply in the case of the elderly patient in rehabilitation, to go home (H05). It is in these declarative statements that there were divergences between the therapist and the patient in the focus of the appraisal. In the following example, the patient expresses his desire to run again [Affect: inclination], a desire which is taken up and positively judged by the therapist.

T. Concerning the symptoms in the foot, we'll monitor them. But probably you'll improve, I think.

P. I have only one [goal], if only I can run again.

T. Yes 

P. But well, I may need to exercise the other toes instead.
T. we didn't talked about that before. It was good you said that cause what's important is the goal,
P. $\quad \mathrm{Mm}$
T. and there you have a clear and obvious ... [goal]. To run again.
P. I'll write it down. Let's go out into the reception (SW1)

Throughout this segment, however, the patient speaks sadly, reflecting his unhappiness about loss of capacity due to chronic back pain. The therapist does not attend to the patient's emotion (his sadness) but adopts the running as a technical goal. Goal setting is commonly used in physiotherapy practice as a way to assess the intervention outcome; the therapist therefore aligns her appraisal to the professional concerns rather than to attending to the patient's emotional statements. When patients introduce emotions, therapists appear hesitant to explore these, prefering to return to a biomechanical focus, as in the following example, in which a patient with back and neck pain introduces her emotions about not knowing (it's nasty) in response to the therapist's question.

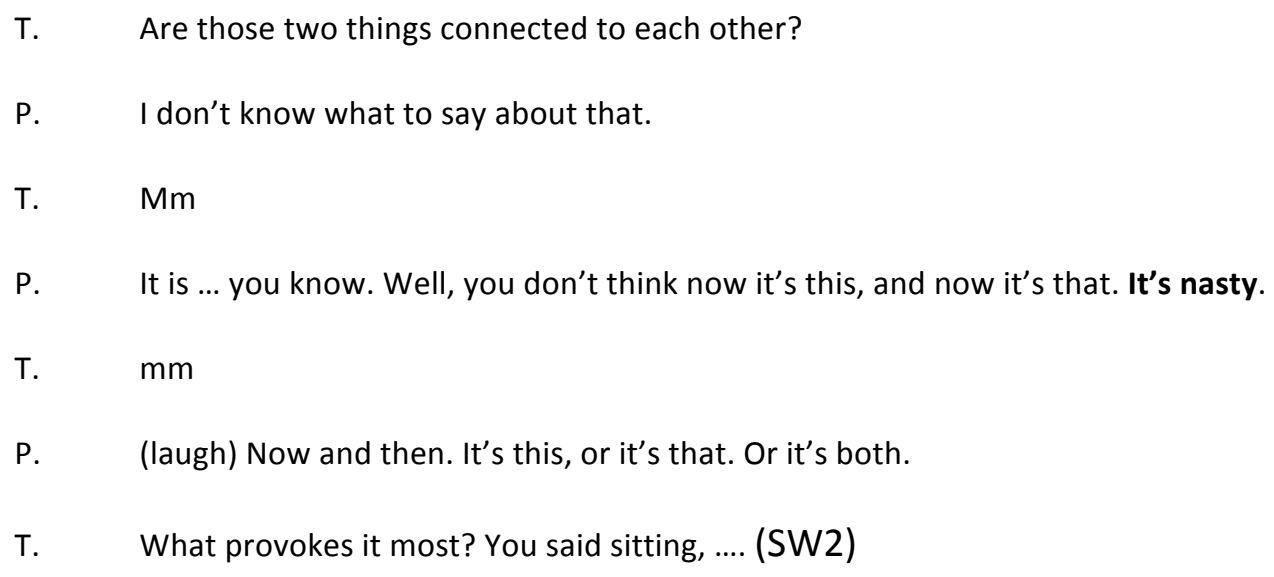

Lack of uptake or acknowledgment of emotional responses by the therapist also occurred in patient Affect appraisals regarding in/security. When patients expressed a confidence with health 
professionals and with people in their neighbourhood who gave advice, therapists tended not to engage with this information:

P. It has started to go down on the ischias-nerve. So now I stretch every morning, try to stretch, and then it becomes a bit better. But it hurts, it really hurts.

T. $\quad$ this stretch, have you discovered it by your-self?

P. I have got tips from my girl-friend. She is engaged in physical exercise. [an extended descriptions of moves]. It helps a bit. It's very stiff.

T. is it mainly leg- and hip-pain that bother you or is it back pain? (SW3)

In the excerpt, the patient gives a rich description of what he does and how, indicating confidence in the girlfriend's competence as advice-giver. The therapist, however, shifts topic and asks about location of the pain. Divergence in and uptake of what was appraised by the two participants was a main finding in the Affect sub-system.

\section{Judgment [behaviours]}

Judgment appraisals were more frequent throughout the consultations than Affect appraisals. They functioned to evaluate the following features: normality, in terms of normal for the general population and for the specific patient; capacity, the patients' physical capacity from a clinical perspective and for tasks of daily living; tenacity, the patient's ability to comply with treatment advice; veracity, the patients' trust in people and also the therapist's trust in the patient; and finally propriety, referring to living a health lifestyle. A key discourse pattern for Judgment of behaviours was the therapists' clinical assessment of a patient's capacity for activities of daily living as well as for sport. Therapists questioned patients about their behaviours as part of their subjective assessment in order to inform decisions about treatment tasks and goals. Patients, as knowledgeable informants of their own capacity, reported their activities. When patients provided 
limited information for the therapist's clinical assessment of capacity, probing questions followed, as in the following example:

T All right... okay... um, all right, and have you been up for

a walk or to the toilet this morning?

$P \quad$ Yes

$T$ Yes?

P Yeah===

THow did you go?

$P \quad$ All right.

$T \quad$ All right?

P Yeah

$T \quad$ Were you tired at all?

$P \quad$ No

$T \quad$ And were you able to do it by yourself? (HO1)

The patient's response, All right provides limited information on his level of fatigue or whether he was able to complete the activity unsupported, hence the therapist's further questioning. In this example, the patient is the appraiser of his functional capacity. Therapists questioned patients' Judgment appraisals for confirmation, Are you sure? (SW1) [Judgment: veracity]; they also appraised the patient's behaviours in terms of normality, that is 'base-line' behaviours, such as such as Stand nice and tall (MS 04) [Judgment: normality +], and Is that when you are slouching (MS 04) [Judgment: normality -].

Another dimension of the Judgment: capacity finding had to do with alignment between therapist and patient, including lack of alignment between the therapist's appraisal and the patient's own of his or her capacity. For example, a patient who described himself as fighting fit when questioned about ambulation, reported that two steps were No problem (HO5). This assessment was at odds 
with the supervisor's evaluation whose treatment goals were independent rather than supported ambulation:

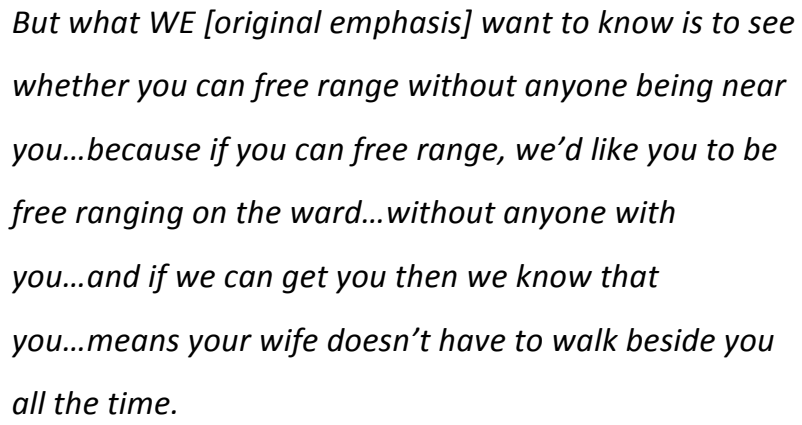

The supervisor's emphasis on we'd like you to be free ranging, that is, the attending health professionals' goals, suggest that these goals take precedence over the patient's goals. In the following example, a similar divergence of alignment is evident when the therapist's evaluation functions to correct the way in which a task designed to assess shortness of breath is carried out. The patient offers her own explanation of why she carries out a task in a particular way, thereby challenging the dominant biomechanical view with her own. The extract illustrates how the patient uses the appraisal resource of Judgment with other linguistic and non-linguistic strategies to contribute her perspective (appraisals are highlighted). The patient is stacking tins as part of a timed assessment task. ( $\mathrm{S}=$ supervisor)

S Next time you don't need to be quite as neat [Judgment: normality -]

T Yep

$S \quad$ It's about doing it as quickly as you can [Judgment: capacity +]

P Oh right

$\mathrm{T}$ You were very neat with how you were pushing them to the side [Judgment: normality - ]

P But that's the way I would do it.

T That's all right, the gentleman before was just... stacking them left right and centre... it was good [Appreciation: valuation + ]

P Okay

$\mathrm{T} \quad$ All right, so... with this next try, l'll give you a bit of a rest 


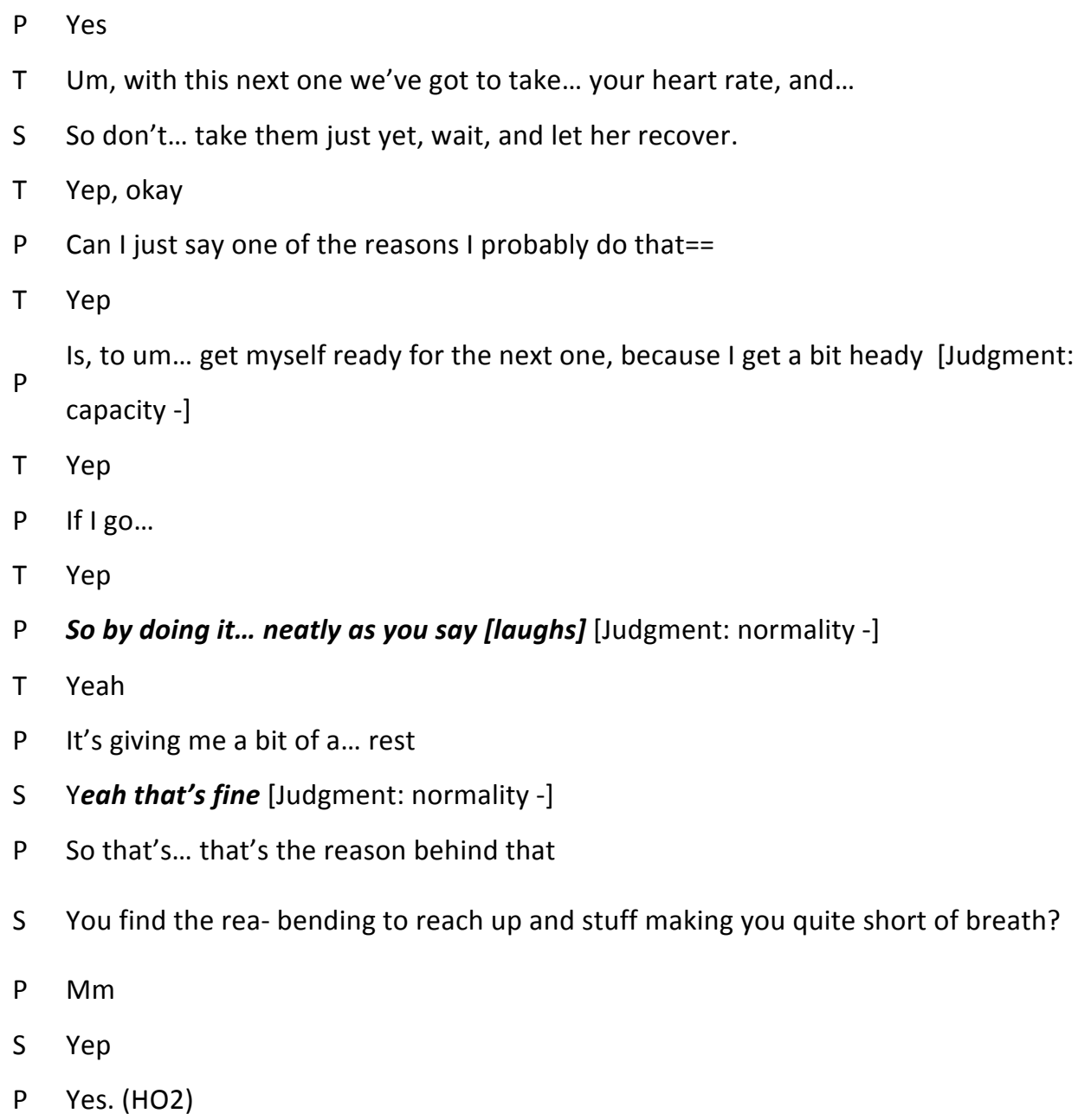

The therapist negatively appraises the patient's efforts as 'neat'. In mounting her defense, the patient adopts a mitigating phrase Can I just say combined with modality probably, with the effect that she does not appear to be questioning the therapist's authority. She provides several reasons in her explanation: One of the reasons... to get myself ready; because I get a bit heady; so by doing it...it gives me a bit of a rest. The patient adopts reported speech, neatly as you say, avoiding aligning herself with the therapist's judgment; again, she mitigates her statement, in this case using humour. The supervisor positively appraises the patient's contribution, Yeah, that's fine [Appreciation], and links the patient's reported strategy to a biomedical explanation, Quite short of breath [Judgment: capacity]. Similarly, patients also self-evaluate their behaviour and performance in terms of 'doing the right thing', for example, One point [Judgment: propriety], 
which a patient humorously comments (HO1) after his therapist's positive evaluation of his performance.

A noteworthy feature of Judgment appraisals is to do with the dimension of what Martin and White (2005) refer to as social-sanction, including Judgment: veracity, and Judgment: propriety, aspects which reflect an ethical dimension of socially sanctioned behaviours. Physiotherapists play an important role in rehabilitating patients who have suffered workplace-related injuries, patients who may be receiving compensation payments. In the 'small talk' opening phase of the consultation one patient reported visiting the snowfields to see snow for the first time with her husband, who is a worker's compensation patient. The patient reports her husband being investigated by his workplace as this activity placed him under suspicion of lying about his workplace-acquired injury. The therapist aligns himself with the patient in his evaluation, expressing his moral objection to the workplace's investigation, you're kidding.

T They had some really good early [snow] falls but um...Do you ever get up to the snow?

P Last year we went.

T Did you ski?

P No. None of us ski, we only went to look I'd never seen snow in my life.

T You're kidding? [Judgment: veracity]

P Nup. So that was just to look. And because with [name] with his leg...

T Mmmm...

P ...he can't do anything. [Judgment: capacity-]

T Right, yeah, you can just imagine him on a snowboard.

P Actually, they enquired at...um...work if he had been on the...you know...did he snowboard, did he ski... [Judgment: propriety]

$\mathrm{T}$ Because they knew he was going up there?

P Yeah, they...someone at work must have said...you know...let them know and he had a big enquiry over it. [Judgment: propriety -]

T You're kidding. [Judgment: veracity -]

$\mathrm{P}$ And because we had had another family and friends with us, he took all the photos into work and said 'here you go', he said 'I've got nothing to hide. [Judgment: propriety] Why would I go 
skiing when I'm nearly crippled'! Huh! [Judgment: capacity]

$\mathrm{T}$ Yeah, there's ways and means of work...you know, enquiring about what their staff are up to but... [Judgment: propriety -]

P Because [name] and, neither of us had seen the snow. So that's what he said, 'I took my family up because they have never ever seen snow.'

$\mathrm{T}$ It's difficult isn't it, when you've got work sort of being accusatory all the time. [Judgment: propriety - ]

The therapist's repetition of you're kidding, and alignment with the patient's Judgment appraisals function to encourage the patient to continue with the anecdote. The alignment of social-sanction appraisals does not appear to contribute to the clinical dimension of the interaction; however, it appears to play an important role in establishing rapport between the patient and therapist.

\section{Appreciation [objects, events]}

The findings for Appreciation in regards to objects which were appraised were pain and body movements, which we interpret as phenomena objectified by the participants. The major events which were appraised concerned patients' reactions to changed pain sensations or bodily movements. The findings for Appreciation show that both therapists and patients drew on this resource to evaluate reactions to tasks, qualities of pain or movements, outcomes of assessment and treatment as well as the value of treatment. As with the findings for Judgment, the focus of the Appreciation was largely a clinical one, with therapists eliciting biomechanical information from the patient in order to make a clinical assessment or conduct a task, for example, about the extent of pain (T) How much does it hurt, on a scale of $0-10$ ? (SW1); the quality of pain (P) It radiates (SW1); location ( $P$ ) It [the back pain] was more isolated (MS6); and severity $(P)$ It's [pain] not as bad though (MS4). As per Judgment appraisals, the Appreciation appraisals were mainly in the context of question-answer exchanges about assessment as well as during examinations, in which the patient's responses guided the therapist's touch. In the following example the therapist 
examines a patient's thoracic spine, standing beside her and pressing his fingers into the painful area:

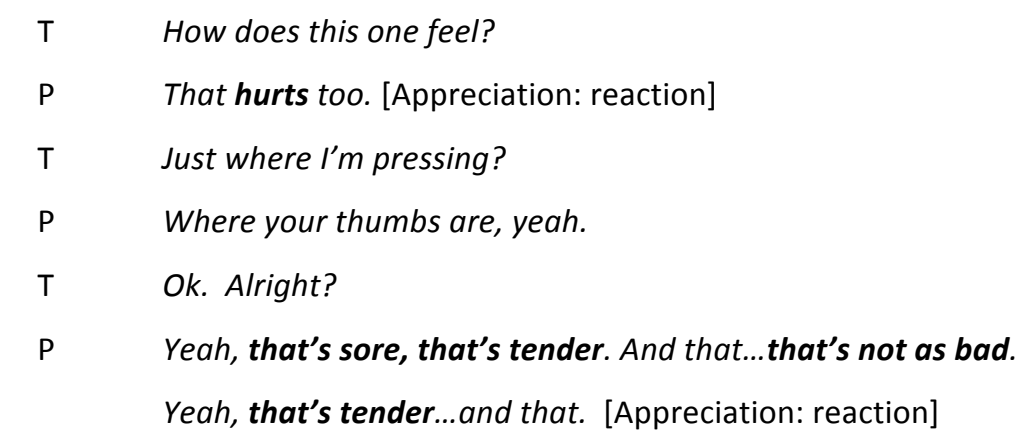

The therapists and patients' Appreciation appraisals of composition in terms of complexity included a shift in focus from a specific body area to a broader understanding of the phenomenon they were encountering: for example, $(T)$ I have an idea about what this is (SW1) in which the therapist indicates a diagnosis for the patient's back pain problem; or $(P)$ The same pain as I'm here for (SW5) in which the patient confirms her understanding of the link between the diagnosis and her current condition.

\section{Alignment}

Throughout the physiotherapy consultations, therapists and patients exchanged evaluations to do with the patients emotions, behaviours, and evaluations of phenomena and processes. Therapists elicited evaluations of patients' overall emotional state at the outset of the consultation and during the physical examination and treatment phase to assess patient well-being and receive permission to continue the examination. This feedback-loop is evident in the exchange-structure (Eggins \& Slade, 1997) pattern of question, response:

$$
\begin{aligned}
& \text { T How are you feeling? } \\
& \mathrm{P} \quad \text { All right. }
\end{aligned}
$$


In the student-patient interactions, this exchange structure was typically followed by a third confirming move in which the therapist repeated the patient's evaluation. Similar microinteractional patterns initiated by the therapist occured in Judgment and Appreciation appraisals in which the focus of the appraisal was bio-mechanical information. In these therapist-initiated exchanges, there is agreement and modification, allowing the therapist to safely continue the examination or treatment. Extended responses from the patient are rare as the unfolding of the discourse tends to be controlled by the therapist. When patients negatively appraised their own behaviours, for example, not completing their exercises, $(P)$ Not as much as I should have been [Judgment: tenacity -] (HO4), their appraisals tended to be aligned with the therapists' treatment goals. These clinical goals are seen to take precedence, as in the rehabilitation example $(T)$ What we'd like you to be is free ranging [Judgment: capacity] (HO5), with the focus primarily on function and evaluating in terms of Appreciation and Judgment, rather than Affect. While patients' do challenge a negative Judgment or Appreciation by the therapist, the alternative view tends to be acknowledged by the therapist, as in the stacking example, but not taken up. These findings suggest that alignment of evaluations allows clinical tasks to be achieved in the physiotherapy consultations; however, the bio-mechanical aspect is foregrounded and that this aspect takes precedence over the patient perspective.

\section{Discussion}

The findings of this study into the language and function of evaluation in physiotherapy consultations show that both patient and therapist attend to evaluation of emotions, behaviours and objects, including objectified pain and biomechanical function. The therapists' and patients' evaluations differ functionally in particular for the Affect sub-system. Judgment and Appreciation 
play a central role in therapists' co-construction of patients' previous physical history and presenting problem to determine a clinical diagnosis and to achieve clinical tasks; in contrast, patients' Affect evaluations, mainly to do with emotions about loss of capacity and pain, are generally acknowledged but not followed up by the therapist. The discourse findings suggest that while patients engage in the consultation from both a clinical and interpersonal perspective, therapists are more focused on their conceptions of what matters clinically.

\section{Evaluative language and the therapeutic relationship}

The findings about the discourse patterns of therapists' evaluations of patient performance build on existing understandings of how the therapeutic relationship is enacted in physiotherapy, and more broadly, about elements of patient-centred care in practice. The findings suggest a potential gap between the espoused values of the physiotherapy profession in regards to patient-centred care and their implementation in practice: while therapists acknowledge their patients' contributions, including emotions as well as insights about performance and treatment goals, they give preference to the bio-mechanical paradigm over the bio-psycho-social. Evidence for this claim is the way in which therapists on the one hand take the interactional initiative when eliciting information about well-being, functional capacity, and decision making about treatment and goals, yet on the other hand, they tend to refrain from evaluating or engaging with certain of the patients' emotions and self-evaluations.

Whilst evaluative language was used by both the therapist and the patient to collaboratively understand and engage with the clinical task, such collaboration did not appear to build the therapeutic relationship in the way in which it has been conceptualized by, for example, Sidani and Fox (2014) who see mutual respect, trust and nurturing as foundational. Instead, the potential of 
the collaborative exchange was limited by the therapists' overarching choice of what and how to evaluate. Our findings contribute a linguistic perspective to existing literature on the dominance of the biomechanical approach among physiotherapists (for instance Thornquist, 2001; Ostelo et al., 2003; Daykin \& Richardson, 2004).

The patients in this study contributed self-assessments in the form of reports about their emotional well-being, how they monitored treatment, and how they provided feedback as well as self-evaluated their performances. While an equitable interaction (Eisenberg, 2012) is seen as a desirable partnership, the discourse patterns of patient self-assessments in this study suggest that there can be a lack of reciprocity and equality in the ways in which the relationship is construed. First, our findings suggest that patients' contributions are not followed up if they do not align with what the therapists' value for clinical tasks and outcomes. One explanation for this apparent dissonance between patients' contributions and therapists' responses may be therapists' discomfort with acknowledging patients' feelings or they may feel patients' contributions can be a threat to their professional expertise (author1 ref et al. 2013). Others have suggested that by not attending to patients' emotions, therapists fail to demonstrate understanding of patients as individuals, and are therapist rather than patient-centred (Cooper et al., 2008; Kidd et al., 2011; Potter et al., 2003). Secondly, patients used humour and mitigating statements in order to bring their self-assessments to the therapists' attention. This finding aligns with previous work in hospital settings that shows patients' use humour and other discourse strategies to counteract the dominant clinical discourse (Eggins, 2014). 


\section{Implications, limitations and future research}

Our study highlights that evaluative language and discourse patterns of what is evaluated, by whom, and the uptake of these evaluations can potentially impact on the reciprocity dimension of the therapeutic relationship. Currently in physiotherapy, the therapeutic relationship is theorised as a component of patient-centred care. In line with Eisenberg's (2012) suggestion for an alternative approach of a more equitable interaction in which the patient is more deeply incorporated, we propose a greater focus on the construct of the therapist-patient relationship in physiotherapy practice and education: this may reduce challenges for therapists connected with the more uni-directional focus on patient-centredness. The clinical implications of how therapists and patients use evaluations, particularly aligned Judgment and Appreciation evaluations, are that they can inform patients' learning (Martin \& Sahlström, 2010). Therapist evaluations can show patients how to move, how not to move, or how to alleviate pain, as part of achieving improved clinical outcomes. On the other hand, therapists' neglect of patients' self-assessments and strategies may hinder achieving treatment goals: research into guides about managing back pain emphasises that supporting patients' self-management strategies can have a better effect than specific treatments (Balagué et al., 2012). The pedagogical implications have to do with raising therapists' awareness of the role of evaluative language in enhancing the relationship with the patient in the clinical interaction. Transcript excerpts of aligned and non-aligned Affect, Judgment, and Appreciation evaluations from this manuscript can be used in discussion with therapists to achieve this aim.

This study has a number of limitations. The participants in this study were not screened in regards to their expertise as patient-centred therapists; therefore the study would benefit from comparing participant perceptions of how they communicate patient-centredness with how they enact these 
views, particularly in regards to evaluation as conceptualised in this study. Patients and therapists were aware the studies were about communication and may have altered their communication. Future studies should ask participants to comment on this aspect once they have been shown the transcripts. We did not attend to the distribution and quantity of evaluation between the study settings, nor did we investigate differences between the more experienced therapists and the students, or take into account therapist and patient gender as a variable. While this was an international study of Swedish and Australian practices, further studies should take these participant variables into account so that the findings can be verified in more heterogeneous physiotherapy settings.

\section{Conclusion}

This discourse analytic study investigated evaluative language in physiotherapy practice. It utilised Appraisal Theory to examine how therapists and patients evaluate patient emotions, behaviours, objects and processes (mainly pain and bodily movements) to contribute to their co-construction of treatment. With its focus on the language used by therapist and patient to contribute their evaluations, this study provides new insights from a social science perspective to inform understandings of clinical practice. 


\section{References}

Australian Physiotherapy Association (2011). Standards for Physiotherapy Practices. ( $8^{\text {th }}$ Ed) Victoria, Australia. Retrieved from https://www.physiotherapy.asn.au/DocumentsFolder/Resources_Private_Practice_Standards_for_ physiotherapy_practices_2011.pdf

Balagué, F., Mannion, A.F., Pellisé, F., \& Cedraschi, C. (2012). Non-specific low back pain. Lancet, 379(9814), 482-491. DOI:10.1016/S0140-6736(11)60610-7

Beeston, S., \& Simons, H. (1996). Physiotherapy practice: practitioners' perspectives. Physiotherapy Theory \& Practice, $12(4), 231-242$.

Besley, J., Kayes, N.M., \& McPherson, K.M. (2011). Assessing therapeutic relationships in physiotherapy: literature review. New Zealand Journal of Physiotherapy, 39(2) 81-91.

Broberg, C., \& Tyni-Lenné, R. (2009). Sjukgymnastik som vetenskap och profession. (Physiotherapy as Science and profession). Stockholm: LSR, Legitimerade Sjukgymnasters Riksförbund.

Chartered Society of Physiotherapy (2014). Quality Assurance Standards. Retrieved from http://www.csp.org.uk/professional-union/professionalism/csp-expectations-members/qualityassurance-standards/section-4-wo

Cooper, K., Smith, B. H., \& Hancock, E. (2008). Patient-centredness in physiotherapy from the perspective of the chronic low back pain patient. Physiotherapy, 94(3), 244-252.

Cruz, E. B., Moore, A., \& Cross, V. (2012). Clinical reasoning and patient-centred care in musculoskeletal physiotherapy in Portugal - A qualitative study. Manual Therapy, 17(3), 246-250.

Daykin, A.R., \& Richardson, B. (2004). Physiotherapists' pain beliefs and their influence on the management of patients with low back pain. Spine, 29(7), 783-795.

Delany, C., (2007). In private practice, informed consent is interpreted as providing explanations rather than offering choices: a qualitative study. Australian Journal of Physiotherapy, 53, 171-177. 
Eggins, S. (2014). Hospital humour: Patient initiated humour as resistance to clinical discourse. In Stracke, E. (Ed). Intersections: Applied Linguistics as a meeting place. (pp. 43-66). Cambridge: Cambridge Scholars Publishing.

Eggins, S. \& Slade, D. (1997). Analysing casual conversation. London: Cassell.

Eisenberg, N.R., (2012) Post-structural conceptualizations of power relationships in physiotherapy. Physiotherapy Theory and Practice, 28(6), 439-446. DOI: 10.3109/09593985.2012.692585.

Halliday, M.A.K. (1978). Language as a social semiotic: The social interpretation of language and meaning. London: Edward Arnold.

Harman, K., Bassett, R., Fenety, A., \& Hoens, A. M. (2011). Client Education: Communicative Interaction between Physiotherapists and Clients with Subacute Low Back Pain in Private Practice. Physiotherapy Canada, 63(2), 212-223.

Hunston, S., \& Thompson, G. (2001). Evaluation in text: Authorial stance and the construction of discourses. New York: Oxford University Press.

Jensen, G. M., Gwyer, J., Shepard, K. F., \& Hack, L. M. (2000). Expert practice in physical therapy. Including commentary by Bartlett, D., Mitchell, R.U. \& Swaine, B.R. with author response. Physical Therapy, $80(1), 28-52$.

Josephson, I., \& Bülow, P. (2014). Utilization of patient resources in physiotherapy interventions: Analysis of the interaction concerning non-specific low back pain. Communication and Medicine. 11(2), 1-000. DOI: $10.1558 /$ cam.v11i2.16694.

Josephson, I., Hedberg, B., \& Bütow, P. (2013). Problem solving in physiotherapy - physiotherapists' talk about encounters with patients with non-specific low back pain. Disability and Rehabilitation. 35(8). 668-677. doi: 10.3109/09638288.2012.705221

Kidd, M. O., Bond, C. H., \& Bell, M. L. (2011). Patients' perspectives of patient-centredness as important in musculoskeletal physiotherapy interactions: a qualitative study. Physiotherapy, 97(2), 154-162.

Martin, C., \& Sahlström, F. (2010). Learning as longitudinal interactional change: From Other-repair to Selfrepair in physiotherapy treatment. Discourse Processes, 47(8), 668-697. 
Martin, J.R. (2000). Beyond Exchange: APPRAISAL Systems in English. In Hunston, S. \& Thompson, G (Eds.) Evaluation in Text: Authorial Stance and the Construction of Discourse. (pp. 142-175) Oxford: Oxford University Press.

Martin, J.R., \& White, P.R.R. (2005). The Language of Evaluation: Appraisal in English. Basingstoke: Palgrave Macmillan.

Mead, N., \& Bower, P. (2000). Patient-centredness: a conceptual framework and review of the empirical literature. Social Science \& Medicine, 51(7), 1087-1110. doi: 10.1016/s0277-9536(00)00098-8.

Mishler, E. G. (1984). Discourse of Medicine: Dialectics of Medical Interviews. Ablex, Norwood, NJ.

Mudge, S., Stretton, C. and Kayes, N. (2014). Are physiotherapists comfortable with person-centred practice? An autoethnographic insight. Disability and Rehabilitation, 36(6): 457-463.

Ostelo, R.W, Stomp-van den Berg, S.G., Vlaeyen, J.W:, Wolters, P.M., \& de Vet, H.C. (2003). Health care providers' attitudes and beliefs towards chronic low back pain: the development of a questionnaire. Manual Therapy, 8(4), 214-222.

Parry, R. (2005). A video analysis of how physiotherapists communicate with patients about errors of performance: insights for practice and policy. Physiotherapy, 91, 204-214.

Pinto, R. Z., Ferreira, M. L., Oliveira, V. C., Franco, M. R., Adams, R., Maher, C. G., \& Ferreira, P.H. (2012). Patient-centred communication is associated with positive therapeutic alliance: a systematic review. Journal of Physiotherapy, 58(2), 77-87.

Potter, M., Gordon, S., \& Hamer, P., (2003). The physiotherapy experience in private practice: The patients' perspective. Australian Journal of Physiotherapy, 49(3), 195-202.

Poulis, I. (2007). Bioethics and physiotherapy. Journal of Medical Ethics, 33(8), 435-436.

Sarangi, S. (2003). Evaluating evaluative language (editorial). Text, 23(2), 165-170.

Sidani, S., \& Fox. M. (2014). Patient-centered care: clarification of its specific elements to facilitate interprofessional care. Journal of Interprofessional Care. 28(2), 134-141 DOI:

$10.3109 / 13561820.2013 .862519$ 
Thornquist, E. (2001). Diagnostics in physiotherapy - processes, patterns and perspectives. Part II. Advances in Physiotherapy, 3(4), 151-162.

Thornquist, E. (1994). Profession and life: separate worlds. Social Science and Medicine. 39, 701-713.

Wade, D. T., \& Halligan, P. W. (2004). Do biomedical models of illness make for good healthcare systems? BMJ: British Medical Journal(7479), 1398. doi: 10.2307/25458519

White, P.R.R. (2004). The Appraisal Website. Retrieved from http://www.grammatics.com/appraisal

Woodward-Kron, R., van Die, D., Webb, G., Pill, J., Elder, C., McNamara, T., \& Manias, E. (2012).

Perspectives from physiotherapy supervisors on student-patient communication. International Journal of Medical Education. 3, 166-174; doi: 10.5116/ijme.502f.6e18. 


\section{University Library}

\section{- M M I N E R VA A gateway to Melbourne's research publications}

Minerva Access is the Institutional Repository of The University of Melbourne

\section{Author/s:}

Josephson, I;Woodward-Kron, R;Delany, C;Hiller, A

Title:

Evaluative language in physiotherapy practice: How does it contribute to the therapeutic relationship?

Date:

2015-10-01

\section{Citation:}

Josephson, I., Woodward-Kron, R., Delany, C. \& Hiller, A. (2015). Evaluative language in physiotherapy practice: How does it contribute to the therapeutic relationship?. SOCIAL SCIENCE \& MEDICINE, 143, pp.128-136. https://doi.org/10.1016/j.socscimed.2015.08.038.

Persistent Link:

http://hdl.handle.net/11343/58554 